\title{
Intensity variations associated with fast sausage modes
}

\author{
M. Gruszecki ${ }^{1}$, V. M. Nakariakov ${ }^{1,2}$, and T. Van Doorsselaere ${ }^{3, \star}$ \\ ${ }^{1}$ Centre for Fusion, Space and Astrophysics, Department of Physics, University of Warwick, Coventry CV4 7AL, UK \\ e-mail: M.Gruszecki@warwick.ac.uk \\ 2 Central Astronomical Observatory at Pulkovo of the Russian Academy of Sciences, 196140 St Petersburg, Russia \\ ${ }^{3}$ Centrum voor Plasma-Astrofysica, Mathematics Department, KULeuven, Celestijnenlaan 200B bus 2400, 3001 Leuven, Belgium
}

Received 28 September 2011 / Accepted 15 March 2012

\begin{abstract}
Aims. We determine the dependence of the observed properties of fast magnetoacoustic axisymmetric oscillations (the sausage mode) of a thick and dense flaring coronal loop, modelled by a magnetic cylinder, on the parameters of the equilibrium plasma configuration. The plasma inside and outside the cylinder is of low-beta, and penetrated by a straight magnetic field. The plasma density has a smooth profile across the magnetic field.

Methods. We use three-dimensional ideal magnetohydrodynamic equations to model numerically the development of the perturbations of the cylindrical equilibrium, considering both leaky and trapped regimes.

Results. Short-period sausage oscillations, trapped by the cylinder, are qualitatively consistent with the analytical results obtained in the models of a plasma slab or a cylinder with a step-function transverse profile. The period of trapped sausage oscillations is determined by the ratio of the phase speed, with the value between the internal and external Alfvén speeds, to the wavelength. Longer-period sausage oscillations are leaky, and their decay times are longer for higher density contrasts between the internal and external media. Leaky sausage oscillations have longer periods than trapped sausage oscillations of the same cylinder. In the coronal conditions, sausage oscillations are essentially compressible and transverse, hence produce modulation of the thermal optically thin emission intensity and periodic Doppler broadening of emission lines. However, if the oscillating plasma non-uniformity is poorly spatially resolved, the variation in the emission intensity is weak and proportional to the actual amplitude of the oscillation squared. The latter variation property is connected with the transverse nature of the oscillation, causing the conservation of mass in the transverse cross-section of the oscillating plasma structure.
\end{abstract}

Key words. magnetohydrodynamics (MHD) - Sun: corona

\section{Introduction}

The magnetically dominated plasma of the solar corona is an elastic and compressible medium that can support the propagation of magnetohydrodynamic (MHD) waves. The structuring of the physical parameters of the corona can dramatically affect the properties of the waves, and this must be taken into account in the interpretation of observed phenomena. A popular model for the study of the effect of the structuring on MHD waves is a straight plasma cylinder, representing, for instance a segment of a coronal loop, prominence tread, plasma jet, plume, etc. A cylinder of dense plasma penetrated by the magnetic field parallel to the axis of the cylinder, and surrounded by a plasma of different density, temperature, and magnetic field, can support the propagation of MHD modes, which are collective perturbations of the internal and external plasma media (e.g. Nakariakov \& Verwichte 2005).

The properties of MHD modes are determined, in particular, by the azymuthal wave number $m$. The axisymmetric fast mode perturbations with $m=0$ are known as the sausage (or radial, or peristaltic) mode. Studies of this mode have attracted attention for many years (Rosenberg 1970; Zaitsev \& Stepanov 1975; Roberts et al. 1984; Cally 1986). This mode is a symmetric perturbation of the cross-section of a plasma non-uniformity, that does not perturb the axis of the loop. The sausage mode is essentially compressive, and the density perturbations are in phase with the perturbations of the magnetic field

* Post-doctoral researcher of the FWO-Vlaanderen. and in anti-phase with the perturbations of the loop minor radius. In the low- $\beta$ plasma typical of solar coronal active regions, the plasma motions induced by the sausage mode are almost perpendicular to the axis of the cylinder. There is also a slow magnetoacoustic mode of the same symmetry, $m=0$, referred to as a slow sausage mode. This mode is mainly longitudinal.

Observationally, the sausage mode has been identified in the microwave and hard X-ray emission produced by flaring coronal loops (Nakariakov et al. 2003; Melnikov et al. 2005; Inglis et al. 2008), in addition to $\mathrm{H}_{\alpha}$ emission from cool, post-flare loops (Srivastava et al. 2008). Owing to the very short periods $(1-10 \mathrm{~s})$ of the observations high time-resolution instruments are required. The first spatially resolved detection of a sausage mode was made by Nakariakov et al. (2003) with the Nobeyama Radioheliograph. They found in-phase variations in the $17 \mathrm{GHz}$ and $34 \mathrm{GHz}$ emission along a thick and short flaring loop, which had a well-pronounced spectral peak near $17 \mathrm{~s}$. The oscillatory signal was strongest at the loop top and had minima near the footpoints. These authors concluded that the fundamental (or global) sausage mode of the flaring loop had been detected. The more detailed studies of Melnikov et al. (2005) confirmed this conclusion. The interest in the coronal fast sausage modes is related to the possibility of a seismological estimate of the external magnetic field in the oscillating plasma non-uniformity, and also the possible role played by this mode in the acceleration of non-thermal particles and their dynamics (see, e.g. Brown \& Hoyng 1975; Zaitsev \& Stepanov 1982). Fujimura \& Tsuneta (2009) reported a sausage mode of the photospheric magnetic 
flux tube that had period in the range from three to six minutes, as observed with Hinode/SOT. Van Doorsselaere et al. (2011) used the detection of the fast sausage mode and a slow mode in a flare to measure the local plasma- $\beta$.

Theoretical studies of the sausage mode of a plasma cylinder were performed in terms of the dispersion relation formalism (Zaitsev \& Stepanov 1975; Edwin \& Roberts 1982; Roberts et al. 1984; Cally 1986; Kopylova et al. 2007). It was pointed out that, depending upon the ratio of the wavelength to the radius of the cylinder, there are two possible regimes, trapped and leaky. Trapped modes are confined to the cylinder, while leaky modes radiate the energy into the external medium. For the typical coronal conditions $(\beta \ll 1)$, trapped sausage modes exist when the external Alfvén speed $C_{\mathrm{Ae}}$ exceeds the value of the internal Alfvén speed $C_{\mathrm{Ai}}$. Even though both fast and slow magnetoacoustic oscillations can have a sausage symmetry, the term "sausage" is usually applied to the fast mode. (Slow sausage modes are usually referred to as "longitudinal", as in the coronal conditions they practically do not perturb the radius of the cylinder.)

The phase speed of the trapped sausage mode is between the Alfvén speed values inside $C_{\mathrm{Ai}}$ and outside $C_{\mathrm{Ae}}$ the plasma structure. In a low- $\beta$ plasma, the condition $C_{\mathrm{Ai}}<C_{\mathrm{Ae}}$ is usually connected with the higher density of the plasma inside the wave-guiding structure. The decrease in the wave number leads to an increase in the phase speed up to the external Alfvén speed, where the mode is subject to a cut-off. For wave numbers smaller than the cut-off value, sausage modes become leaky, owing to the violation of the total internal reflection condition. Additional leakage can be associated with the loop curvature (see, e.g. Verwichte et al. 2006), but we do not consider this possibility in this study. The cut-off value for the wave number decreases with the increase in the density contrast between the internal and external media (see, e.g. Roberts et al. 1984). In numerical studies of the zero- $\beta$ limit, Pascoe et al. (2007b) demonstrated that leaky global sausage modes can exist in long loops with small density contrasts. The period of the leaky global sausage mode was found to be almost independent of the internal Alfvén speed $C_{\mathrm{Ai}}$ and determined by the Alfvén speed outside the loop $C_{\mathrm{Ae}}$. Additionally, it was shown that the period of the global sausage mode of a thick and dense plasma cylinder, which is typical of for instance a flaring coronal loop, was determined by the loop length $L$ (Nakariakov et al. 2003; Aschwanden et al. 2004). The numerical studies of Pascoe et al. (2007a, 2009) confirmed that this result is insensitive to both the internal structuring of the oscillating cylinder and the variation in its cross-sectional area in the longitudinal direction. Inglis et al. (2009) also confirmed the results obtained by Pascoe et al. (2007b) for finite plasma $\beta$ and additionally showed that the period of the global sausage mode is insignificantly affected by finite values of $\beta$, with the variation in remaining less than $5 \%$ for a broad range of plasma parameters. The latter statement was applied to the normalised value of the period, when the effect of the modification of the equilibrium values was not considered. Thus, it was concluded that the period of the sausage oscillations of typical flaring loops, with a large aspect ratio of the minor radius to the length, is determined by the loop length rather than its minor radius.

The use of the slab model instead of the cylinder for the study of sausage oscillations is usually justified by the apparent consistency among the analytical results obtained for those geometries in the trapped regime (Edwin \& Roberts 1983; Roberts et al. 1984). However, there is a significant difference in the behaviour of the waves outside the plasma non-uniformity in the cartesian and cylindrical geometries, as pointed out by, e.g. Díaz et al. (2004).

The aim of this paper is to study the observational properties of trapped and leaky sausage modes of a thick and dense flaring loop in the thermal, optically thin emission, accounting for the effects of cylindrical geometry. The properties of the sausage mode are studied by direct numerical simulations of the initial value problem, with the use of the full set of ideal MHD equations, in the three-dimensional geometry. We choose a cylinder with smooth radial (transverse) profiles of the equilibrium physical quantities. In contrast to the case of a plasma slab, which allows for a simple analytical solution of the linear problem in the case of the zero- $\beta$ plasma for a symmetric Epstein profile (see, e.g. Cooper et al. 2003b), for a cylindrical geometry, analytical solutions are known only for the stepfunction transverse profiles (Edwin \& Roberts 1983). However, even in this case, the analytical study of leaky modes is rather non-trivial, as it requires a careful treatment of different branches of the Bessel functions of a complex argument (see Ruderman \& Roberts 2006, for detail). Thus, our approach is similar to the modelling performed by Terradas et al. (2007), who studied the excitation of the fast magnetoacoustic modes of a plasma cylinder. However, the main emphasis of our work is on the forward modelling of the observational manifestation of the sausage modes. Modelling placed sausage oscillations in terms of the initial value problem, as applied in this work, is adequate for the aim of this study which are to study the observational manifestation of the response of a coronal loop to an impulsive axisymmetric excitation. As in thick and dense flaring loops, the ratio of the minor radii to the lengths is rather large, the analytical results obtained in the thin flux tube approximation are not quantitatively applicable. In this study, we do not investigate the dependence of the damping time of leaky modes on the physical parameters of the equilibrium. The reason is that in the available observations (e.g. Melnikov et al. 2005; Kopylova et al. 2007; Inglis et al. 2008) sausage oscillations are not subject to damping, as their lifetime is limited by the duration of observations (i.e. the gyrosynchrotron emission from the loop). Moreover, for the detection of an oscillation the primary parameter is the period, not its damping time. An apparent decrease in the oscillation amplitude can indeed be connected not only with damping, but also with the modification of the observational conditions.

This paper is organised as follows. The analytical model and numerical methods are described in Sect. 2. Numerical results are presented and discussed in Sect. 3, and the observability of the sausage mode is discussed in Sect. 4. This paper concludes with a summary of the main results in Sect. 5.

\section{Numerical setup}

To model a sausage oscillation of a plasma cylinder, we used the ideal magnetohydrodynamic (MHD) equations

$$
\begin{aligned}
& \frac{\partial \varrho}{\partial t}+\nabla \cdot(\varrho \boldsymbol{V})=0, \\
& \varrho \frac{\partial \boldsymbol{V}}{\partial t}+\varrho(\boldsymbol{V} \cdot \nabla) \boldsymbol{V}=-\nabla p+\frac{1}{\mu}(\nabla \times \boldsymbol{B}) \times \boldsymbol{B}, \\
& \frac{\partial \boldsymbol{B}}{\partial t}=\nabla \times(\boldsymbol{V} \times \boldsymbol{B}), \\
& \frac{\partial p}{\partial t}+\boldsymbol{V} \cdot \nabla p=-\gamma p \nabla \cdot \boldsymbol{V}, \\
& \nabla \cdot \boldsymbol{B}=0,
\end{aligned}
$$


where $\varrho$ is the mass density, $p$ is the gas pressure, $\boldsymbol{B}$ is the magnetic field, $\boldsymbol{V}=\left[V_{x}, V_{y}, V_{z}\right]$ is the flow velocity, $\mu$ is the magnetic permeability and $\gamma=5 / 3$ is the ratio of the specific heats. In this study, we ignore the effects of gravity, field-line curvature, and twist.

We modelled a coronal loop as a straight magnetic cylinder. The equilibrium density profile was given by the Gaussian function:

$\varrho_{0}(x, y)=\left(\varrho_{\mathrm{i}}-\varrho_{\mathrm{e}}\right) \exp \left(-\frac{x^{2}+y^{2}}{2 w^{2}}\right)+\varrho_{\mathrm{e}}$,

where $\varrho_{\mathrm{i}}$ is the density at the loop centre, $\varrho_{\mathrm{e}}$ is the density of the ambient medium far from the loop, and $w$ is the half-width of the Gaussian function. The equilibrium magnetic field was assumed to be uniform in the $z$-direction, but in the transverse direction given by the profile

$B_{z}(x, y)=\left(B_{\mathrm{i}}-B_{\mathrm{e}}\right) \exp \left(-\frac{x^{2}+y^{2}}{2 w^{2}}\right)+B_{\mathrm{e}}$.

The internal value of the magnetic field $B_{\mathrm{i}}$ was lower than the external value $B_{\mathrm{e}}$. We assumed that initially the cylinder is in a pressure balance with the surrounding plasma, and that at the beginning the plasma flows are zero in the simulation region. To maintain the total pressure balance between the cylinder and surrounding plasma, we chose the gas pressure within the cylinder according to the condition

$p_{\mathrm{i}}=\frac{1}{2 \mu}\left(B_{\mathrm{e}}^{2}-B_{\mathrm{i}}^{2}\right)+p_{\mathrm{e}}$.

We defined the Alfvén speed $C_{\mathrm{A}}=B / \sqrt{\mu \rho}$ and the sound speed $C_{\mathrm{s}}=\sqrt{\gamma p / \rho}$ in the usual way. The Alfvén speed outside the cylinder was higher than inside it, which is the necessary condition for the existence of the trapped modes.

Equations (1)-(5) are numerically solved with the use of the Lagrangian-remap code Lare3d (Arber et al. 2001). In our studies, we simulated the plasma dynamics in a domain $(-10,10) \times$ $(-10,10) \times(0,7) \mathrm{Mm}$ covered by $400 \times 400 \times 100$ grid points. We performed grid convergence studies to check the numerical results. At the footpoints of the cylinder, we implemented linetying boundary condition to mimic the dense layer of the solar atmosphere. Elsewhere, we set zero-gradient boundary conditions. The specific spatial scales shown above are given just for reference, and can be readily changed by re-normalisation.

To excite a sausage mode, we used a pulse in the transverse components of the velocity

$V_{x 0}=A x \exp \left(-\frac{x^{2}+y^{2}}{2 w^{2}}\right) \sin (k z)$,

$V_{y 0}=A y \exp \left(-\frac{x^{2}+y^{2}}{2 w^{2}}\right) \sin (k z)$,

where $k=\pi N / L_{0}$ is the longitudinal wave number with $N$ being the longitudinal mode number, and $A$ is an initial amplitude, and $L_{0}$ is the length of the cylinder. Choosing different values of the integer $N$, we excited different standing longitudinal harmonics, with the lowest being in the leaky regime. To avoid nonlinear effects, we set $A=0.01 C_{\mathrm{Ae}}$. For small values of $\beta$ this amplitude can be comparable with the sound speed. However, as for small $\beta$ the sausage mode is essentially transverse, it is mainly determined by the values of the Alfvén speed in the equilibrium, which was confirmed by our experiments.
In all our experiments, the ratio of the cylinder width $w$ to its length was kept constant, 2/7. In different experiments, we changed the density contrast between the internal and external plasmas from 2 to 10 . The ratio of the internal to external values of the magnetic field was kept constant, 0.89. The difference between the temperatures of the internal and external plasmas was varied to keep the total pressure balance. The value of plasma- $\beta$ was always lower than 0.3 . Hence, in our experiments we studied plasma cylinders with the ratio of the external to internal Alfvén speeds from 1.41 to 3.16 .

\section{Spatial structure and periods of sausage modes}

We performed a series of numerical experiments simulating the sausage mode in the magnetic cylinder. For this, we excited perturbations of the equilibrium configuration, induced by the pulse described in the previous section. The initial perturbation developed into a trapped or leaky global sausage mode oscillation, depending upon the excited longitudinal harmonic (as the ratio of the longitudinal wavelength to the width of the cylinder for different harmonics is either greater or smaller than the cut-off value). Figure 1 shows contour plots of the perturbations of the mass density $\varrho$, the longitudinal component of the magnetic field $B_{z}$, a transverse component of the velocity $V_{y}$, and the longitudinal component of the velocity $V_{z}$ in the plane $y=0$ at the time $t=3 w / C_{\mathrm{Ae}}$ for the fifth harmonic of the sausage mode. It is clearly visible that the axis of the cylinder is not perturbed. Perturbations of the mass density and magnetic field are in phase, while the radius of the cylinder is perturbed in anti-phase with the density and magnetic field. Owing to the finite value of plasma- $\beta$ (the maximum value in the cylinder is $\beta=0.3$ ), longitudinal flows are present in the cylinder, but its amplitude is about an order of magnitude smaller than for the transverse flows.

Figure 2 shows a snapshot of the profile of the perturbation of density versus the transverse coordinate $x$ in a sausage mode, obtained from the simulation (the blue curve). We can see that the mode rearranges the plasma in the cylinder in the transverse direction, causing the density enhancements and rarefactions.

Qualitatively, this behaviour is extremely consistent that the estimates that can be obtained analytically for a cylinder with a cylindrical coordinate system $(r, \varphi, z)$ and a discontinuous density profile

$\varrho_{0}(r)= \begin{cases}\varrho_{\mathrm{i}} & \text { for } r<w, \\ \varrho_{\mathrm{e}} & \text { for } r>w,\end{cases}$

as described in e.g. Edwin \& Roberts (1983). Using a Fourier decomposition in time and $z(\sim \exp i(k z-\omega t))$, the solutions with azimuthal symmetry $(\partial / \partial \varphi=0)$ or sausage modes can be described with the equations

$\frac{\mathrm{d}^{2} P^{\prime}}{\mathrm{d} r^{2}}+\frac{1}{r} \frac{\mathrm{d} P^{\prime}}{\mathrm{d} r}-\kappa^{2} P^{\prime}=0$,

$\rho\left(\omega^{2}-k^{2} C_{\mathrm{A}}^{2}\right) V_{r}=-\mathrm{i} \omega \frac{\mathrm{d} P^{\prime}}{\mathrm{d} r}$,

$\rho\left(\omega^{2}-k^{2} C_{\mathrm{A}}^{2}\right) V_{\phi}=0$,

$\rho\left(\omega^{2}-k^{2} C_{\mathrm{T}}^{2}\right) V_{z}=\omega k \frac{C_{\mathrm{s}}^{2}}{C_{\mathrm{s}}^{2}+C_{\mathrm{A}}^{2}} P^{\prime}$, 

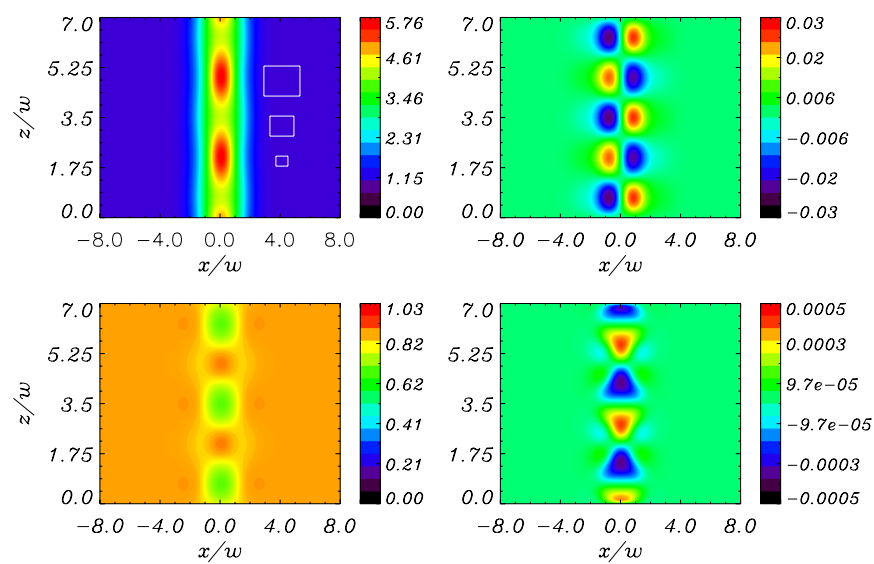

Fig. 1. Snapshots of the perturbations of the physical parameters in a plasma cylinder at $t=3$ (the time is normalised to $w / C_{\mathrm{Ae}}$ ): the mass density $\varrho$ (top left panel), the longitudinal component of the magnetic field $B_{z}$ (bottom left panel), the transverse component of the velocity $V_{y}$ (top right panel), and the longitudinal component of the velocity $V_{z}$ (bottom right panel). The spatial coordinates are measured in units of $w$ that is the half width of the cylinder.

where $P^{\prime}$ is the total pressure perturbation, $\kappa^{2}=$ $\frac{\left(k^{2} C_{\mathrm{s}}^{2}-\omega^{2}\right)\left(k^{2} C_{\mathrm{A}}^{2}-\omega^{2}\right)}{\left(C_{\mathrm{s}}^{2}+C_{\mathrm{A}}^{2}\right)\left(k^{2} C_{\mathrm{T}}^{2}-\omega^{2}\right)}, C_{\mathrm{T}}^{2}=C_{\mathrm{s}}^{2} C_{\mathrm{A}}^{2} /\left(C_{\mathrm{s}}^{2}+C_{\mathrm{A}}^{2}\right)$, and $\omega$ is the solution of the dispersion relation

$\frac{\kappa_{\mathrm{e}}}{\varrho_{\mathrm{e}}\left(k^{2} C_{\mathrm{Ae}}^{2}-\omega^{2}\right)} \frac{K_{0}^{\prime}\left(\kappa_{\mathrm{e}} w\right)}{K_{0}\left(\kappa_{\mathrm{e}} w\right)}=\frac{\sqrt{-\kappa_{\mathrm{i}}^{2}}}{\varrho_{\mathrm{i}}\left(k^{2} C_{\mathrm{Ai}}^{2}-\omega^{2}\right)} \frac{J_{0}^{\prime}\left(\sqrt{-\kappa_{\mathrm{i}}^{2}} w\right)}{J_{0}\left(\sqrt{-\kappa_{\mathrm{i}}^{2}} w\right)}$,

where $K_{0}$ and $J_{0}$ are the zeroth order Bessel functions. In this system, the density perturbation can be calculated from

$\varrho_{\text {pert }}=\varrho-\varrho_{0}=-\frac{\mathrm{i}}{\omega} \nabla \cdot\left(\varrho_{0} \boldsymbol{V}\right)$.

The solution for the discontinuous equilibrium density profile is shown together with the numerical solution in Fig. 2. The solutions follow qualitatively the same behaviour. The discontinuity in the density perturbation for the idealised cylinder is of course smoothed out in the numerical solution. Thus, in a low- $\beta$ environment, the plasma is redistributed mainly in the transverse direction, while the total mass of a certain cross-section remains almost the same (and exactly the same in the zero- $\beta$ case). This can also be inferred from Eqs. (13) and (15), where the vertical velocity component is clearly proportional to the plasma- $\beta$, and the horizontal component is not. We also checked in the simulations that the longitudinal contribution $\partial V_{z} / \partial z$ to the divergence of velocity $\nabla \cdot \boldsymbol{V}$ is smaller than the perpendicular contribution $\partial V_{x} / \partial x+\partial V_{y} / \partial y$.

Figure 3 shows two typical scenarios for the time evolution of the sausage modes: the trapped (top panel) and leaky (bottom panel) regimes. The trapped mode corresponds to the fifth longitudinal harmonic and the leaky mode to the first harmonic. Hence, their wavelengths are either greater or smaller than the cutoff wavelength, respectively. The signal shown is the perturbation of the longitudinal component of magnetic field, $B_{z}$, at the centre of cylinder. Because of their symmetry, both excited modes undergo the maximum perturbation of this physical quantity there. The signal corresponding to the trapped mode is almost harmonic and decay-less. The apparent variations in its amplitude are caused by some minor contamination of the signal by other harmonics that are excited because of the mismatch

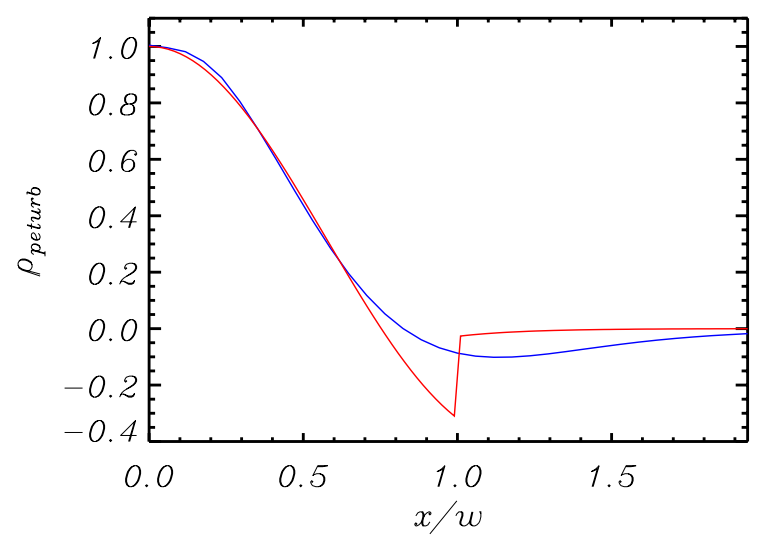

Fig. 2. Snapshots of the density perturbation as a function of the radial coordinate. The blue line corresponds to the result obtained by simulations for a plasma cylinder, and the red line shows the solution in the cylinder with a discontinuous density profile.

between the initial excitation and the spatial structure of the fifth longitudinal harmonics. In the leaky regime, we see that the oscillation is also harmonic, but decays quickly with time. The decay is associated with the radiation of the fast magnetoacoustic waves in the external medium. The efficiency of the leakage is determined by the ratio of the longitudinal wavelength to the cutoff wavelength. In physical terms, for a fixed wavelength and the diameter of the cylinder, the leakage is more efficient for a lower ratio of the internal to external densities. In Fig. 3, we illustrate this effect by showing the time behaviour of the leaky mode for two values of the density contrast, $\varrho_{\mathrm{i}} / \varrho_{\mathrm{e}}=10$ (black line) and $\varrho_{\mathrm{i}} / \varrho_{\mathrm{e}}=5$ (red line). It is evident that leaky sausage oscillations are damped more slowly in a denser cylinder. These results are in an agreement with Cally (1986), Cally (2003), Smith et al. (1997), Nakariakov et al. (2003) and Terradas et al. (2005) who showed that the wave leakage is weaker in a denser loop. In addition, there is some indication of transient shorter period oscillations, which may be connected with the higher leaky harmonics (Cally 1986, 2003). These modes decay very quickly and are hardly detectable in real data in the presence of noise.

\section{Manifestation of sausage modes in observations}

The sausage mode is essentially compressible, producing a modulation of the density of the plasma and the absolute value of the magnetic field. On the other hand, in a low- $\beta$ plasma, this mode is also essentially transverse, causing plasma flows mainly in the direction perpendicular to the magnetic field. From the essential compressibility of this mode, it is often deduced that it must be responsible for significant perturbations in the intensity of the emission generated in the oscillating object. However, as pointed out in Cooper et al. (2003a), the manifestation of MHD modes in observations in the optically thin regime is also significantly affected by the effects of the line-of-sight (LOS) integration namely the change in the column depth of the waveguide, along the LOS, by the mode. In particular, the importance of this effect for the sausage mode arguably observed in the modulation of gyrosynchrotron emission, was pointed out by Fleishman et al. (2008). We consider the role of the LOS-integration effect for the thermal emission.

In the zero- $\beta$ regime, the sausage mode only moves the plasma in the direction across the magnetic field (see Eq. (15)). Hence, according to the mass conservation law, the mass of a transverse cross-section of the oscillating cylinder should always 

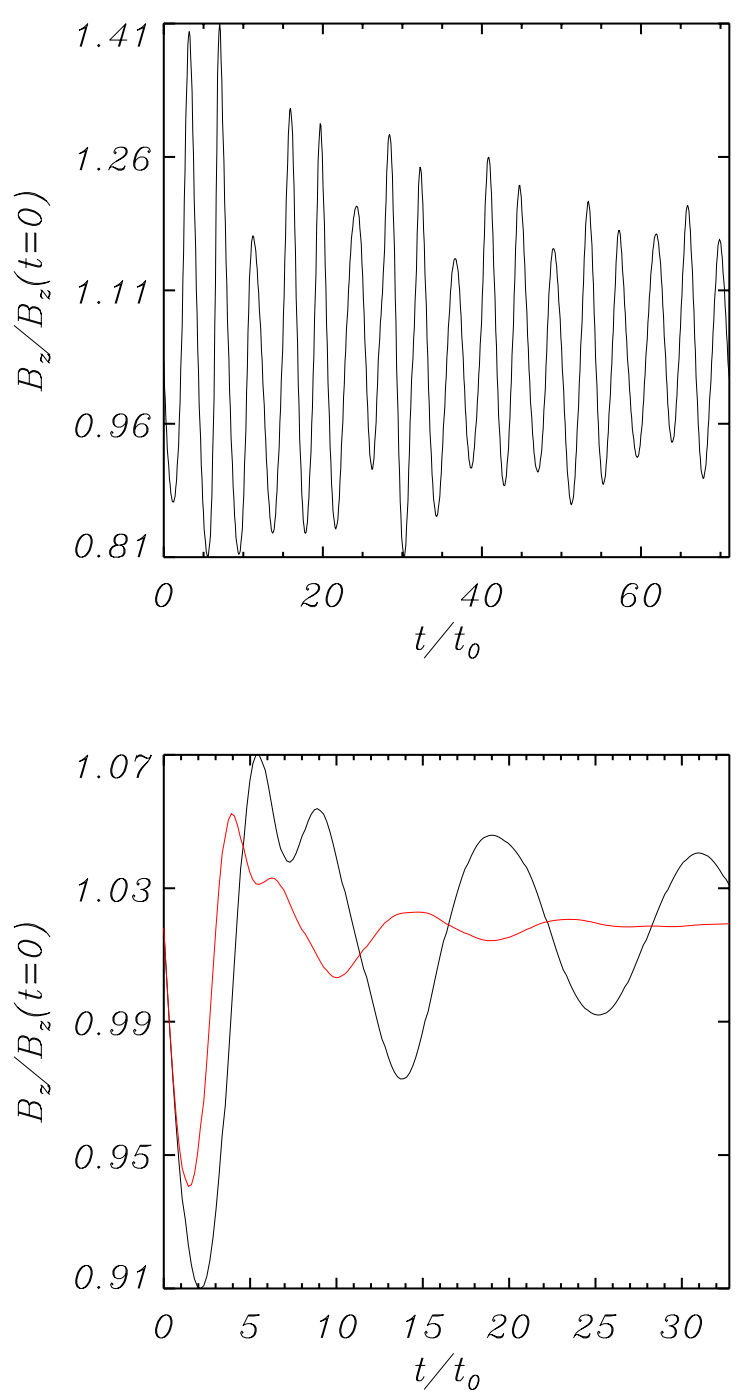

Fig. 3. Time dependence of the longitudinal component of the magnetic field, $B_{z}$ at the centre of the cylinder for the fifth (top panel) and first (bottom panel) longitudinal harmonics of the sausage mode, corresponding to trapped and leaky modes, respectively. The black curve in the bottom panel corresponds to the density contrast of 10 , and the red curve to 5 .

remain constant during the oscillation. If one were to probe the mass in the cylinder, by integrating the mass density along an infinitely thin LOS, the "LOS mass" would vary in time, as the plasma moves not only along the LOS but also sideways. On the other hand, if the "width" of the LOS (in other words, the pixel size or the half-width of the point spread function) were larger than the diameter of the waveguiding cylinder, the displaced mass would always be in the region of the integration and hence the integral remain constant in all phases of the oscillation cycle.

This effect is illustrated by Fig. 4. Using the results of the numerical simulations described in Sect. 3, we estimated the integral of the density along the $x$-axis at the centre of the cylinder for different widths $\Delta S_{\text {pixel }}$ of the LOS

$\mathcal{M}(t)=\iint_{\Delta S_{\text {pixel }}} \mathrm{d} S \int_{x \text { along LOS }} \varrho(\boldsymbol{r}, t) \mathrm{d} x$,

where $\mathrm{d} S=\mathrm{d} y \mathrm{~d} z$ is an infinitesimal area element in the planeof-sky. The specific value of the LOS width was controlled by the number of the computational grid points, and was measured
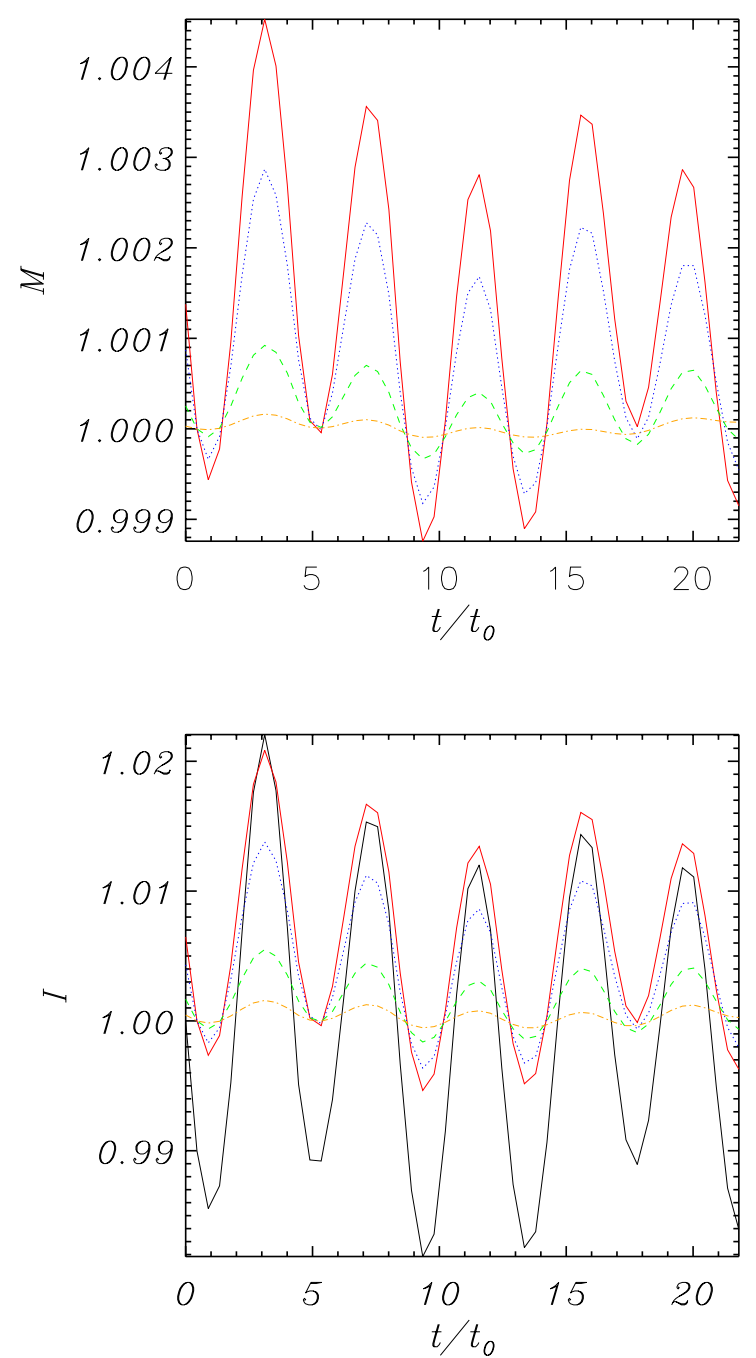

Fig. 4. The time dependence of the LOS-integrated mass $\mathcal{M}$ (top panel) and emission intensity $\mathcal{I}$ (bottom panel) in a sausage-oscillating cylinder for different "widths" of the LOS (pixel sizes). In all cases, the LOS is directed across the cylinder and passes through its centre. The red (solid) lines correspond to an infinitely thin line-of-sight, the blue (dotted) lines correspond to the pixel with the size of the half width of the cylinder $w$, the green (dashed) lines to $2 w$ and the orange (dasheddotted) lines to $3 w$ lines, respectively. The black solid line in the bottom panel shows the relative variation in the density at the centre of the cylinder. The time is measured in the units $t_{0}=w / C_{\mathrm{Ae}}$.

in the units of the equilibrium width $w$ of the cylinder. The pixels of the different sizes, used in the calculations, are shown in the top left panel of Fig. 1. It is evident from Fig. 4 that for sufficiently large pixels, perturbations of the LOS-integrated mass tends to zero.

In the optically thin regime, thermal emission is proportional to the density squared. Hence, the emission intensity can be estimated as

$\mathcal{I}(t)=\iint_{\Delta S_{\text {pixel }}} \mathrm{d} S \int_{x \text { along LOS }} \varrho^{2}(\boldsymbol{r}, t) \mathrm{d} x$.

The time variation in the emission intensity for different sizes of the pixel are shown in the bottom panel of Fig. 4. For reference, we give the relative perturbation of the density at the centre of the cylinder. It is evident that the increase in the pixel size leads to a sharp decrease in the relative amplitude of the emission intensity, as in the case of the LOS-integrated mass. This can be 
readily understood as for perturbations of a weak amplitude, the relative density squared can be taken as

$$
\begin{aligned}
\mathcal{I}(t)= & \iint_{\Delta S_{\text {pixel }}} \mathrm{d} S \int_{x} \varrho^{2} \mathrm{~d} x \\
= & \iint_{\Delta S_{\text {pixel }}} \mathrm{d} S \int_{x}\left(\varrho_{0}+\varrho_{\text {pert }}\right)^{2} \mathrm{~d} x \\
= & \iint_{\Delta S_{\text {pixel }}} \mathrm{d} S \int_{x}\left(\varrho_{0}^{2}+2 \varrho_{0} \varrho_{\text {pert }}+\varrho_{\text {pert }}^{2}\right) \mathrm{d} x \\
= & \mathcal{I}_{0}+\iint_{\Delta S_{\text {pixel }}} \mathrm{d} S \int_{x}\left(\left[\left(\varrho_{\mathrm{e}}+\left(\varrho_{\mathrm{i}}-\varrho_{\mathrm{e}}\right) f(r)\right] \varrho_{\text {pert }}\right.\right. \\
& \left.+\varrho_{\text {pert }}^{2}\right) \mathrm{d} x \\
= & \mathcal{I}_{0}+\varrho_{\mathrm{e}} \iint_{\Delta S_{\text {pixel }}} \mathrm{d} S \int_{x} \varrho_{\text {pert }} \mathrm{d} x \\
& +\left(\varrho_{\mathrm{i}}-\varrho_{\mathrm{e}}\right) \iint_{\Delta S_{\text {pixel }}} \mathrm{d} S \int_{x} f(r) \varrho_{\text {pert }} \mathrm{d} x \\
& +\iint_{\Delta S_{\text {pixel }}} \mathrm{d} S \int_{x} \varrho_{\text {pert }}^{2} \mathrm{~d} x,
\end{aligned}
$$

where $f(r)$ is a smooth (preferably monotonic) function that describes the radial variation $\left(r^{2}=x^{2}+y^{2}\right)$ in the density with the extra conditions $f(0)=1$ and $\lim _{r \rightarrow \infty} f(r)=0$. In the expression for the intensity, three terms are affected by time variability (through $\varrho_{\text {pert }}$ ). The first term (Eq. (20)) is $\mathcal{M}(t)$. It is exactly zero when the pixel size $\Delta S_{\text {pixel }}$ becomes large, because it just expresses the conservation of mass. We numerically checked that the term given by Eq. (21) becomes increasingly small when $\Delta S_{\text {pixel }}$ becomes large. This only leaves the last term (Eq. (22)) as the main source of variability in the intensity, which is of the second order in the oscillation amplitude. Hence, the time variation in the intensity is quadratically proportional to the density perturbation and negligibly small.

We only considered a line-of-sight that is perpendicular to the loop axis. Investigating the effect of the angle of the line-of-sight and the loop axis will be the subject of future research.

\section{Summary and discussion}

We have performed a numerical study of fast magnetoacoustic oscillations of a thick and dense coronal loop, modelled by a plasma cylinder embedded in a plasma with different properties and penetrated by a straight magnetic field, emphasising their observational appearance. We considered only the oscillations of the sausage symmetry (with the azimuthal wave number $m=0)$. The plasma both inside and outside the cylinder was taken of low- $\beta$. The transverse profile of the plasma density was smooth, without any steep gradients or discontinuities. The oscillations were impulsively excited and then simulated with the use of the full set of ideal MHD equations. Only oscillations with low amplitudes were considered.

The behaviour of short-wavelength sausage oscillations of a plasma cylinder is well closely with the analytical results obtained for a cylinder with a step-function profile (Zaitsev \& Stepanov 1975; Edwin \& Roberts 1983; Roberts et al. 1984; Cooper et al. 2003b). The oscillations are trapped in the cylinder, their phase speed has a value between the Alfvén speed inside and outside the cylinder, and their period is determined by the ratio of the phase speed to the wavelength. Sausage waves are essentially compressible, perturbing the density of the plasma and the absolute value of the magnetic field, and transverse, moving the plasma in the direction across the field, when the plasma- $\beta$ is sufficiently low.

Sausage oscillations of wavelengths longer than the cutoff value are leaky. The attenuation of the oscillations depends on the density contrast between the cylinder and the ambient medium, which is qualitatively consistent with the analytical prediction made in Cally $(1986,2003)$. For the plasma parameters typical of coronal plasma structures, e.g. dense plasma loops, the damping time can be of several oscillations periods. Thus, long-wavelength sausage modes can last for a sufficiently long time to before being detected in observations.

Incidentally, we have numerically shown that the mechanism of resonant absorption does not operate for the axisymmetric sausage modes (Goossens et al. 1992). Although the simulation included a smooth transition layer, where in principle a resonance could occur between the global mode and localised Alfvén continuum modes, no resonant behaviour has been found in the numerical results. The sausage modes are damped only by MHD radiation (in the leaky regime), and not by resonant absorption.

The observational manifestation of sausage oscillations in the thermal emission, whose strength is proportional to the density of the emitting plasma squared, depends upon whether the oscillating plasma non-uniformity is spatially resolved. If the pixel size or the half-width of the spread function are about the width of the non-uniformity, the perturbation of the emission intensity by sausage oscillations is proportional to the relative amplitude squared. For example for an oscillation of the amplitude $5 \%$, the observed amplitude of the intensity variation is only $0.25 \%$. This effect should be taken into account when distinguishing between the sausage oscillations and, e.g., torsional oscillations, which both produce periodic non-thermal broadening of the emission lines by the Doppler shift. We would like to emphasise this funding: an essentially compressible fast sausage oscillation does not cause variations in the optically thin emission intensity of a detectable level when the diameter of the oscillating plasma cylinder is insufficiently resolved.

We conclude that the sausage mode is a good tool for the seismological probing of the plasma in oscillating coronal plasma non-uniformities, giving us information about the Alfvén speed inside and outside the non-uniformity. The observational manifestation of this mode is very much affected by the LOS-integration effect and the pixel size, and it has to be taken into account in the data analysis.

Acknowledgements. The authors thank the referees for stimulating comments. M.G. is supported by the Newton International Fellowship NF090143. T.V.D. has received funding from the Odysseus programme of the FWO-Vlaanderen and from the EU's Framework Programme 7 as an ERG with grant number 276808 .

\section{References}

Arber, T. D., Longbottom, A. W., Gerrard, C. L., \& Milne, A. M. 2001, J. Comp. Phys., 171, 151

Aschwanden, M. J., Nakariakov, V. M., \& Melnikov, V. F. 2004, ApJ, 600, 458 Brown, J. C., \& Hoyng, P. 1975, ApJ, 200, 734 
Cally, P. S. 1986, Sol. Phys., 103, 277

Cally, P. S. 2003, Sol. Phys., 217, 95

Cooper, F. C., Nakariakov, V. M., \& Tsiklauri, D. 2003a, A\&A, 397, 765

Cooper, F. C., Nakariakov, V. M., \& Williams, D. R. 2003b, A\&A, 409, 325

Díaz, A. J., Oliver, R., Ballester, J. L., \& Roberts, B. 2004, A\&A, 424, 1055

Edwin, P. M., \& Roberts, B. 1982, Sol. Phys., 76, 239

Edwin, P. M., \& Roberts, B. 1983, Sol. Phys., 88, 179

Fleishman, G. D., Bastian, T. S., \& Gary, D. E. 2008, ApJ, 684, 1433

Fujimura, D., \& Tsuneta, S. 2009, ApJ, 702, 1443

Goossens, M., Hollweg, J. V., \& Sakurai, T. 1992, Sol. Phys., 138, 233

Inglis, A. R., Nakariakov, V. M., \& Melnikov, V. F. 2008, A\&A, 487, 1147

Inglis, A. R., Van Doorsselaere, T., Brady, C. S., \& Nakariakov, V. M. 2009, A\&A, 503, 569

Kopylova, Y. G., Melnikov, A. V., Stepanov, A. V., Tsap, Y. T., \& Goldvarg, T. B. 2007, Astron. Lett., 33, 706

Melnikov, V. F., Reznikova, V. E., Shibasaki, K., \& Nakariakov, V. M. 2005 A\&A, 439, 727

Nakariakov, V. M., \& Verwichte, E. 2005, Liv. Rev. Sol. Phys., 2, 3
Nakariakov, V. M., Melnikov, V. F., \& Reznikova, V. E. 2003, A\&A, 412, L7

Pascoe, D. J., Nakariakov, V. M., \& Arber, T. D. 2007a, Sol. Phys., 246, 165

Pascoe, D. J., Nakariakov, V. M., \& Arber, T. D. 2007b, A\&A, 461, 1149

Pascoe, D. J., Nakariakov, V. M., Arber, T. D., \& Murawski, K. 2009, A\&A, 494, 1119

Roberts, B., Edwin, P. M., \& Benz, A. O. 1984, ApJ, 279, 857

Rosenberg, H. 1970, A\&A, 9, 159

Ruderman, M. S., \& Roberts, B. 2006, Sol. Phys., 237, 119

Smith, J. M., Roberts, B., \& Oliver, R. 1997, A\&A, 317, 752

Srivastava, A. K., Zaqarashvili, T. V., Uddin, W., Dwivedi, B. N., \& Kumar, P. 2008, MNRAS, 388, 1899

Terradas, J., Oliver, R., \& Ballester, J. L. 2005, A\&A, 441, 371

Terradas, J., Andries, J., \& Goossens, M. 2007, Sol. Phys., 246, 231

Van Doorsselaere, T., De Groof, A., Zender, J., Berghmans, D., \& Goossens, M. 2011, ApJ, 740, 90

Verwichte, E., Foullon, C., \& Nakariakov, V. M. 2006, A\&A, 449, 769

Zaitsev, V. V., \& Stepanov, A. V. 1975, A\&A, 45, 135

Zaitsev, V. V., \& Stepanov, A. V. 1982, Sov. Astron. Lett., 8, 132 